\title{
Reflexive Arbeitskräftewirtschaft und Mitbestimmung in der TV-Content-Produktion
}

Die Mitbestimmung der Beschäftigten in Betrieb und Unternehmen setzt stabile Belegschaften und Betriebsstrukturen - auch in räumlicher Hinsicht - voraus. Das Personalmanagement ist als Ansprechpartner für die Interessenvertretung von zentraler Bedeutung. Ohne kompetente Ansprechpartner auf der Arbeitgeberseite ist eine effektive Partizipation an den Entscheidungen des Personalmanagements nicht möglich. Was passiert mit der Mitbestimmung der Arbeitnehmer, wenn sich dieser Akteur im Zuge eines Wandels der Praxis des Personalmanagements in Richtung reflexiver Arbeitskräftewirtschaft verflüchtigt und wenn Belegschaften destabilisiert werden sowie Arbeitsorte wechseln? Am Beispiel empirischer Ergebnisse aus der TV-Content-Produktion können die Konsequenzen schon heute studiert werden.

\section{Vom Strategic Human Resource Management zur Arbeitskräftewirtschaft}

Eher verwaltende Praktiken des Personalmanagements werden vor dem Hintergrund der Wettbewerbsverschärfung in den 1980er Jahren durch ein Strategic Human Resource Management abgelöst, das die Unternehmens- und Personalstrategie stärker verzahnt und Personalmanagement weiter ökonomisiert (Staehle 1989). Der moderne Management- und Netzwerkdiskurs erweitert in den 1990er Jahren die Perspektive, indem er auch die Interorganisationsbeziehungen von Unternehmungen in die Formulierung und Implementation von Personalstrategien einbezieht. Als Erster hat Werner Nienhüser (1999) sich mit diesen neuen Praktiken des Personalmanagements auseinandergesetzt. Da klassische Analysen zum Personalmanagement diese Perspektivenerweiterung nur ungenügend erfassen, wird deshalb in diesem Beitrag auch der von Nienhüser (1999, S. 168) eingeführte Begriff der Arbeitskräftewirtschaft für den Zugriff auf Arbeitskräfte verwendet. Er bezieht sich auf das Zusammenspiel von Arbeitskräften in Organisationen mit denen in interorganisationalen Beziehungszusammenhängen.

Das bundesdeutsche Mitbestimmungssystem ist auf die mit der Arbeitskräftewirtschaft verknüpfte Perspektivenerweiterung relativ schlecht vorbereitet, denn es ist hauptsächlich auf eine Belegschaft in einem Betrieb an einem Ort ausgerichtet, die hierarchisch koordiniert wird (Kißler et al.
2011). Gleichwohl verbreiten sich Praktiken einer Arbeitskräftewirtschaft in einer Reihe von Branchen bzw. „organisationalen Feldern“ (DiMaggio/Powell 1983, S. 148). Dies gilt u. a. für bestimmte projektbasierte Industrien wie die Bauindustrie (Nienhüser 1999) oder die IT-Industrie (Barley/ Kunda 2004). Über Leiharbeit (Promberger 2006), „Arbeitskräftepools“ (Weinkopf 1996), über kollegiale Arbeitnehmerüberlassung (Hertwig 2009), die Zusammenarbeit mit Freiberuflern und über Subunternehmerketten verbreiten sich Praktiken der Arbeitskräftewirtschaft auch in den Kernsektoren der Wirtschaft. Besonders ausgeprägt ist die Arbeitskräftewirtschaft in der Kultur- und Medienindustrie (Haak 2008). Man kann dort und insbesondere in der Fernsehproduktion, der TV-ContentProduktion, schon seit einiger Zeit die „Zukunft in der Gegenwart erfahren“ (Windeler et al. 2001). Aus diesem Grund liegt den folgenden Ausführungen auch eine Analyse der Praktiken der Arbeitskräftewirtschaft in dieser Branche zugrunde.

Eine umfassende Analyse der Praktiken der Arbeitskräftewirtschaft und ihrer Konsequenzen für die Mitbestimmung ist wegen ihrer Komplexität in diesem Beitrag nicht möglich. Ich frage deshalb am Beispiel der Arbeitskräfteselektion, wie die Praktiken der Mitbestimmung aussehen und wer mit welchen Rechten und wie weitgehend daran partizipieren kann. Die Ausführungen beschränken sich auf die Arbeitskräfteselektion, weil sie (nicht nur) in der TV-Content-Produktion wegen der Dominanz der Koordinationsform „Projektnetzwerk" höchst bedeutsam ist und dementsprechend - wie wir im empirischen Teil der Ausführungen sehen werden - auch durch Medienkonzerne beeinflusst wird. Sie gehört zudem zum Kernbereich der Mitbestimmung. Ferner lässt die Analyse dieser „personalwirtschaftlichen Funktion" Veränderungen in den Praktiken und in der Akteurskonstellation besonders deutlich werden.

In diesem Aufsatz zeige ich, dass herkömmliche Mitbestimmungspraktiken nahezu irrelevant werden und dass andere Akteure mit unterschiedlich weitreichenden Partizipationsmöglichkeiten an die Stelle von Betriebsräten treten. Trotzdem existieren Ansatzpunkte für einen Ausbau der Mitbestimmung der Arbeitskräfte, ohne jedoch eine Angleichung an die Praktiken in den Kernindustrien jemals zu erreichen.

Meine Ausführungen basieren zum einen auf der Sekundärauswertung von mehr als 80 leitfadengestützten Interviews mit Akteuren aus der Fernsehindustrie. Im Zuge der Auswertung dieses empirischen Materials zeigte sich jedoch, dass sich die Art und Weise des Zugriffs auf Arbeitskräfte von dem in Kernsektoren der Wirtschaft unterscheidet. Deshalb wurde

\footnotetext{
1 Erhebung im Rahmen des von der Deutschen Forschungsgemeinschaft (DFG) finanzierten Forschungsprojekts „Vernetzte Content-Produktion für das digitale Fernsehen“" Projektleitung: Jörg Sydow und Arnold Windeler, zwischen 1997 und 2001. Wenn aus diesen Interviews zitiert wird erhalten die Bezeichnungen der Interviewpartner ein "a".
}

Carsten Wirth, Dr., ist Professor für Verwaltung und Netzwerkarbeit in der Sozialwirtschaft an der Hochschule Kempten. Arbeitsschwerpunkte: Arbeit, Organisation und Netzwerke in und jenseits der Sozialwirtschaft. e-mail: carsten.wirth@fh-kempten.de 
eine Follow-up-Untersuchung durchgeführt, die die Arbeitskräftewirtschaft in der TV-Content-Produktion analysiert. ${ }^{2}$ Diese Studie ermöglicht also die Berücksichtigung von Veränderungen im Zeitablauf, z. B. die Veränderungen durch die Medienkrisen in den Jahren 2001 und 2008. Auch ihre Ergebnisse fließen in die folgende Abhandlung ein.

Die Daten wurden in der zweiten Studie wieder mit leitfadengestützten Interviews mit Akteuren aus der Fernsehindustrie erhoben und mit Bezug zu zentralen strukturationstheoretischen Kategorien (grundlegend dazu Giddens 1984) ausgewertet. In diesem Projekt wurden in eineinhalb bis sechs Stunden dauernden Interviews ein Branchenexperte (Branchenexperte 1), drei Vertreter von Fernsehproduzenten (Produzent 1, 2, 3), ein Vertreter von einem Berufsverband (Berufsverband 1) und ein Vertreter der Vereinten Dienstleistungsgewerkschaft (ver.di 1) befragt. Nach Abschluss des Erhebungsprozesses wurden die Forschungsergebnisse an den Teil der Interviewpartner rückgemeldet, die für eine Kommentierung der Ergebnisse zur Verfügung standen, und es wurden Divergenzen in den Interpretationen diskutiert. In zwei Fällen schrieben die befragten Personen auch kurze Gutachten (Gutachten 1, 2), nicht zuletzt um neuere Entwicklungen einzubringen.

Mein Argument entwickle ich wie folgt: In Abschnitt 3 stelle ich in gebotener Kürze die Grundlagen der Mitbestimmung bei der Arbeitskräfteselektion vor. Abschnitt 4 geht auf die Besonderheiten von Arbeit in der TV-Content-Produktion ein und erläutert, welche Mitbestimmungspraktiken bei der Arbeitskräfteselektion existieren und welche Akteure relevant sind. AbschlieBend gehe ich in Abschnitt 5 auf mögliche Ansatzpunkte ein, um die Mitbestimmung der Arbeitskräfte zu stärken. Zuvor jedoch erläutere ich in den Begriff der reflexiven Arbeitskräftewirtschaft (Abschnitt 2).

\section{Reflexive Arbeitskräfte- wirtschaft: Begriff, funktionale und Querschnittsprobleme}

Werner Nienhüser (1999) stellt in seiner Studie der Bauwirtschaft die Markt-Subkontrahierung einer Netzwerk-Subkon- trahierung und einer damit assoziierten netzwerkbezogenen Arbeitskräftewirtschaft gegenüber. Durch Praktiken einer Arbeitskräftewirtschaft erweitert sich der Fokus auf den markt- und netzwerkförmig koordinierten Beziehungszusammenhang einer Organisation sowie auf das Feld, in das die Organisation sozial eingebettet ist. Unter Arbeitskräftewirtschaft verstehe ich den organisierten Zugriff auf Arbeitskräfte einschließlich der Arbeitskräfte in netzwerkförmigen Beziehungszusammenhängen mit Subunternehmungen und Freiberuflern oder in dominant vom Preis gesteuerten marktlichen Beziehungen. Die Beziehungsqualität, also der hierarchische, marktliche oder netzwerkförmige Zugriff auf Arbeitskräfte, sowie deren Wandel ist Gegenstand der Überlegungen. Zudem nehmen die Akteure im Zuge einer Arbeitskräftewirtschaft auf umfassendere Kontexte, z. B. das organisationale Feld und die Gesellschaft, gezielt Einfluss, genauso wie Feld und Gesellschaft die Praktiken der Arbeitskräftewirtschaft prägen. Das hat u. a. zur Konsequenz, dass Praktiken einer Arbeitskräftewirtschaft durch Industriespezifika beeinflusst werden. Dementsprechend gibt es auch keinen one best way der Arbeitskräftewirtschaft, weil sich die Praxis der Arbeitskräftewirtschaft im rekursiven Zusammenspiel von branchen-, netzwerk- und unternehmungsbezogenen Praktiken konstituiert.

Wenn die Akteure, die in die Arbeitskräftewirtschaft involviert sind, systematisch und kontinuierlich Daten erheben, aufbereiten, auswerten und in ihrem Handeln verwerten, spreche ich von ,reflexiver Arbeitskräftewirtschaft" (Wirth 2010, S. 13). Der Grad der Reflexivität kann dabei variieren. Trotz vorhandener Reflexivität gelingt nicht alles (unintendierte Handlungsfolgen) oder die Akteure agieren vor dem Hintergrund unerkannter Handlungsbedingungen, die zuweilen sogar das Produkt unintendierter Handlungsfolgen sind (Giddens 1984, S. 5).

Mit jeder Form reflexiver Arbeitskräftewirtschaft müssen drei übergeordnete Querschnittsprobleme gelöst werden: das Effektivitäts-, das Effizienz- und das Herrschaftssicherungsproblem. Mit der Lösung des Effektivitätsproblems wird sichergestellt, dass grundlegende Ziele von Unternehmungen oder des Netzwerkes erreicht werden, z. B. das langfristige Überleben am „Markt“. Die Lösungen für das Effizienz- problem gewährleisten, dass die Arbeitskräftewirtschaft wirtschaftlich erbracht wird. Dies betrifft einzelne Funktionen, z. B. die Arbeitskräfteselektion, wie auch das Zusammenspiel unterschiedlicher Funktionen, z. B. der Arbeitskräfteselektion mit der Arbeitskräfteentwicklung. Mit den Lösungen für das Herrschaftssicherungsproblem wird eine Ordnung legitimiert, die Privilegien für einzelne Akteure bereithält und Abhängigkeiten anderer auf Dauer stellt.

Die Lösungen dieser grundlegenden Probleme der Arbeitskräftewirtschaft sind an Aktivitäten im Zuge der Arbeitskräftewirtschaft gebunden bzw. an die Praktiken der Lösung der funktionalen Probleme der Arbeitskräftewirtschaft gekoppelt. Indem eine Arbeitskräfteversorgung einer Branche sichergestellt wird und eine Arbeitskräfteplanung, die Arbeitskräfteselektion, eine Arbeitskräftebindung, der Arbeitskräfteeinsatz, eine Arbeitskräfteentwicklung, eine Koordination der Arbeitskräfte und eine Arbeitskräftefreistellung betrieben wird, lösen die Akteure die funktionalen Probleme der Arbeitskräftewirtschaft. Die Art und Weise, wie diese funktionalen Probleme der Arbeitskräftewirtschaft gelöst werden, prägt die Lösung der Querschnittsprobleme, wie deren jeweils etablierte Lösung die Bearbeitung der funktionalen Probleme beeinflusst.

Die Bedeutung interorganisationaler Zusammenhänge für die Lösungen der Probleme der Arbeitskräftewirtschaft kann z.B. branchenspezifisch, für spezifische Funktionen und im Zeitablauf variieren. In der TV-Content-Produktion erfolgen die Problemlösungen z.B. vorwiegend in interorganisationalen Beziehungen (Wirth 2010). Allerdings erfolgt in der TVContent-Produktion die Arbeitskräftekoordination vor allem durch Beschäftigte. Die Arbeitskräfteentwicklung hingegen ist nahezu vollständig in interorganisationale Beziehungszusammenhänge, an die Arbeitskräfte und an das organisationale Feld ausgelagert (Wirth 2010, S. 113ff.). Der Bedeutungszuwachs der Leiharbeit, z.B. in der Metall- und Elektroindustrie, seit den Hartz-Reformen (Crimmann et al. 2009) ist ein Beispiel für die Veränderung der Bedeutung interorganisationaler Bezie-

2 Projekt „Arbeitskräftewirtschaft und Arbeitsregulation in Projektnetzwerken am Beispiel der Fernsehproduktion“, gefördert von der HansBöckler-Stiftung in den Jahren 2007 und 2008 
hungen in der Arbeitskräftewirtschaft im Zeitverlauf. Darüber hinaus variiert die Bedeutung umfassenderer Kontexte wie organisationaler Felder für die Arbeitskräftewirtschaft. Das organisationale Feld hat für die Arbeitskräfteentwicklung in der TV-Content-Produktion eine zentrale Bedeutung (Wirth 2010, S. 113ff.). Dieses ist in anderen Branchen von geringerer Bedeutung, da der Nachwuchs vorwiegend intern ausgebildet wird.

Wenn sich die jeweiligen Praktiken der reflexiven Arbeitskräftewirtschaft als gangbar erweisen, also den Interessen, Sichtweisen und Legitimationsgrundlagen der jeweiligen Anspruchsgruppen genügen (dazu ausführlich Ortmann 1995, S. 367ff.), tragen die Akteure zur Reproduktion der Praktiken bei und prägen diese zugleich. Damit ist das, was als wirtschaftlich gilt, nicht das Ergebnis technologischer oder markt- und produktionsökonomischer Erfordernisse, sondern ist auch Ausdruck kognitiver, normativer und machtbezogener Strukturen und dementsprechend kontingent, d.h. auch anders möglich, ohne jedoch beliebig zu sein.

In die Arbeitskräftewirtschaft sind unterschiedliche Akteure involviert. Dazu können auch betriebliche Interessenvertretungen gehören. Ich gehe deshalb auf die Mitbestimmungsrechte von Betriebsräten im Prozess der Arbeitskräfteselektion ein.

\section{Partizipation im Prozess der Arbeitskräfteselektion}

Unter Partizipation verstehe ich die unterschiedlich weitgehende Beeinflussung von Entscheidungen, in diesem Beitrag der Arbeitskräfteselektion, die individuell und/oder kollektiv erfolgen kann (ähnlich Kißler et al. 2011, S. 25ff.). Von einer kollektiven Partizipation spreche ich, wenn ein Zusammenschluss von zwei oder mehr Akteuren an einer Entscheidung partizipiert. Letzteres kann z.B. durch demokratisch gewählte betriebliche Interessenvertretungen erfolgen. Dieser kollektive Partizipationsbegriff ist aber auch offen für die Nutzung von Beziehungszusammenhängen, z.B. von „schwachen Beziehungen" (Granovetter 1973), im Prozess der Personalauswahl. Dann partizipiert eine kleine Gruppe von Personen an der Arbeitskräfteselektion. Insofern ist das hier zugrunde gelegte Verständnis von Kollektivität gradueller Art.

Im Zuge individueller Partizipation beeinflusst der Bewerber in Abhängigkeit von seiner individuellen Ressourcenausstattung (z.B. Knappheit der eigenen Qualifikation) den Ausgang des Bewerbungsverfahrens. Eine kollektive Partizipation über Betriebsräte kann in $10 \%$ aller Betriebe mit ca. $45 \%$ aller Beschäftigten in West- und $37 \%$ aller Beschäftigten in Ostdeutschland erfolgen (Ellguth/Kohaut 2011, S. 245). Diese Betriebsräte verfügen über eine Reihe von Mitbestimmungsrechten bei der Arbeitskräfteauswahl, die vor dem Hintergrund der jeweiligen „Interaktionskultur“ (König 2005) im Betrieb zur Anwendung kommen. Neben der innerbetrieblichen Ausschreibung nach $₫ 93$ Betriebsverfassungsgesetz (BetrVG) und den Auswahlrichtlinien nach $\$ 95$ BetrVG ist in Unternehmen mit mehr als 20 Arbeitnehmern das Widerspruchsrecht gegen die Einstellung eines bestimmten Bewerbers zentral, weil der Betriebsrat mit Widersprüchen den internen Arbeitsmarkt auch machtvoll mitgestalten und das Management in Aushandlungsprozesse zwingen kann (König 2005).

Eine Partizipation der Betriebsräte an Auswahlentscheidungen in interorganisationalen Beziehungszusammenhängen ist nur im Ausnahmefall vorgesehen. Handelt es sich bei Formen selbstständiger Erwerbstätigkeit wie z.B. von freien Mitarbeitern tatsächlich jedoch um eine Beschäftigung von Arbeitnehmern, so greifen die oben genannten Mitbestimmungsrechte. Bei Leiharbeitnehmern findet das Mitbestimmungsrecht bei Einstellungen gemäß $\$ 14$ Absatz 3 Arbeitnehmerüberlassungsgesetz (AÜG) Anwendung. Allerdings zeigen empirische Analysen, dass sowohl bei Formen scheinselbstständiger Erwerbstätigkeit (Wirth 1999) als auch bei Leiharbeitern (Promberger 2006) Mitbestimmungsrechte kaum zur Anwendung kommen oder nicht ausgeschöpft werden.

Die Ausführungen zeigen, dass die Mitbestimmung rechtlich und praktisch weitgehend auf den „Normalbetrieb“ ausgerichtet ist. Wie verändern sich diese Praktiken und die Akteurskonstellation, wenn diese Voraussetzung nicht mehr erfüllt ist?

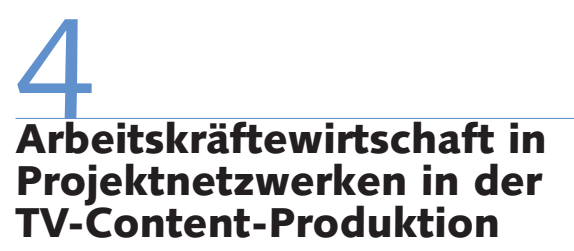

\subsection{ARBEIT IN PROJEKTNETZWERKEN}

In der TV-Content-Produktion haben sich Projektnetzwerke als dominante Koordinationsform in einem historischen Prozess durchgesetzt (zusammenfassend Wirth 2010, S. 48ff.). Sie bestehen aus zeitlich befristeten, auf Projekte bezogenen Geschäftsbeziehungen und -interaktionen, die am Projekt beteiligte Unternehmungen projektbezogen, aber eben auch projektübergreifend unterhalten und mit Bezug zum Beziehungszusammenhang, eben netzwerkförmig, koordinieren. Die Akteure koordinieren in Projektnetzwerken ihre jeweiligen zeitlich befristeten Projektaktivitäten unter Rückgriff auf Erfahrungen aus vorhergehenden Projekten sowie unter Vorwegnahme einer möglichen Zusammenarbeit in zukünftigen Projekten. In dem rekursiven Zusammenspiel projektbezogener und projektübergreifender Koordination liegt die entscheidende Ursache dafür, dass es sich bei Projektnetzwerken um mehr als bloß temporäre Systeme handelt (Sydow/Windeler 1999).

Die projektübergreifenden Beziehungen erlangen über den Mechanismus der Aktualisierung und Institutionalisierung eine gewisse Stabilität: Akteure aktualisieren im projektbezogenen Handeln Praktiken, Interaktionen und Beziehungen aus früheren Projekten, z.B. indem sie wiederkehrend mit den gleichen Subunternehmungen und Freiberuflern zusammenarbeiten. Erst dadurch werden sie in die Lage versetzt, komplexe und risikoreiche Projekte fristgerecht und im Budget zu bewältigen. Durch ihren Gebrauch im Projekt werden die Praktiken als anerkannte Praktiken des Projektnetzwerks institutionalisiert und bei weiteren Projekten aufgegriffen. Dabei ist die Selektion von Unternehmungen und von Arbeitskräften von zentraler Bedeutung, da hierüber die Reproduktion der Praktiken relativ weitgehend gesichert werden kann (Windeler et al. 2000).

In Projektnetzwerken in der fiktionalen TV-Content-Produktion sind konzerngebundene Fernsehsehsender und -produzenten die wichtigsten Koordina- 
Übersicht 1: Unterschiede von Arbeit im „Normalbetrieb“ und in Projektnetzwerken in der TV-Content-Produktion

\begin{tabular}{|c|c|c|}
\hline & Arbeit im "Normalbetrieb" & $\begin{array}{l}\text { Arbeit in Projektnetzwerken der } \\
\text { TV-Content-Produktion }\end{array}$ \\
\hline Koordinationsform & Hierarchie & (Projekt-) Netzwerk \\
\hline Größe des Betriebs & $\begin{array}{l}\text { Wenige Großbetriebe, vor allem } \\
\text { KMU's }\end{array}$ & Kleinstbetriebe \\
\hline Dauer der Zusammenarbeit & langfristig & kurzfristig \\
\hline Arbeitsort & Betrieb & wechselnd \\
\hline Belegschaft & stabil & instabil \\
\hline Status & Arbeitnehmer/in & $\begin{array}{l}\text { Wechsel zwischen selbstständiger } \\
\text { und abhängiger Erwerbstätigkeit }\end{array}$ \\
\hline Karrieren im & internen Arbeitsmarkt & (Projekt-) Netzwerk \\
\hline Arbeitsmarktstruktur & Rand- und Stammbelegschaft & $\begin{array}{l}\text { segmentierte Arbeitskräftepools } \\
\text { rechtlich selbstständiger } \\
\text { Unternehmungen }\end{array}$ \\
\hline
\end{tabular}

toren. Deshalb sind Projektnetzwerke in dieser Branche häufig „konzerngesteuerte Projektnetzwerke“ (Wirth/Sydow 2004). Dies hat - wie wir noch sehen werden auch Konsequenzen für die Arbeitskräfteselektion, die im Konzernzusammenhang, insbesondere vom Fernsehsender beim Fernsehproduzenten, systematisch beeinflusst wird.

Die zeitlich befristete, aber wiederkehrende Arbeit in Projekten in Projektnetzwerken in der TV-Content-Produktion unterscheidet sich von „Normalarbeit“ in Großbetrieben der Industrie oder des Dienstleistungssektors, die hierarchisch koordiniert wird. Übersicht 1 gibt einen Überblick:

Die Phase der konkreten Zusammenarbeit ist relativ kurz. Für das Produktionspersonal in der Produktion eines TV-Films wie einer Tatort-Folge beträgt die Drehzeit ca. 20 Arbeitstage. Sie erfolgt an unterschiedlichen Orten. Zudem wandelt sich die Zusammensetzung des Pro- duktionspersonals von Projekt zu Projekt, sodass eine stabile Belegschaft nicht entsteht. Die Arbeitskräfte verändern - oft aus eigenem Antrieb - ihren Status: In einem Projekt arbeiten sie als Selbstständige, im nächsten als Arbeitnehmer. Sie orientieren sich bei ihrer Karriereentwicklung nicht an internen Arbeitsmärkten von Betrieben, sondern an Projektnetzwerken und deren produkt- und funktionsspezifischen Aufstiegswegen. Die Auftraggeber und die Arbeitskräfte bringen eine Segmentation der Arbeitskräfte in Stamm- und Randarbeitskräfte in den Pools von Arbeitskräften der Fernsehproduktionsunternehmungen und ihren Subunternehmungen hervor (ausführlicher Windeler/Wirth 2004, S. 307). Damit unterscheidet sich Arbeit in Projektnetzwerken in der TV-ContentProduktion hinsichtlich zentraler Charakteristika von Arbeit in „Normalbetrieben“ und macht eine Mitbestimmung über Betriebsräte nahezu unmöglich, weil diese u.a. auf eine stabile Belegschaft angewiesen

\section{Abb. 1: Selektion von Arbeitskräften in der TV-Content- Produktion}

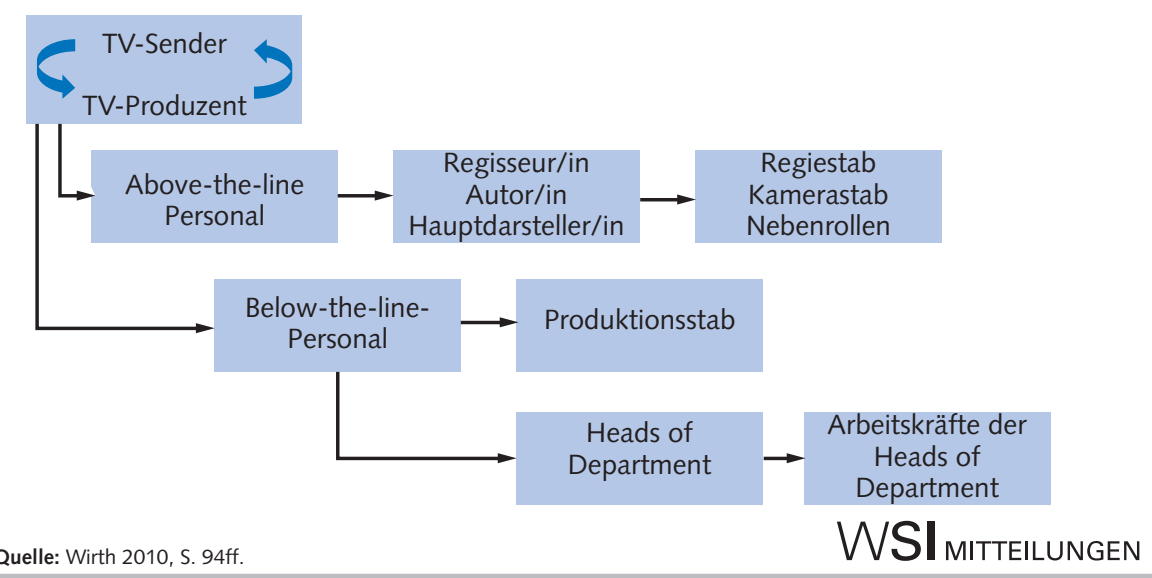

ist. Dementsprechend finden sich kollektive Mitbestimmungspraktiken, wie wir sie z.B. aus der Metall- und Elektroindustrie kennen, nur in wenigen und kleiner werdenden „Inseln traditioneller Regulation“ (Windeler/Wirth 2004, S. 308), den öffentlich-rechtlichen Fernsehsendern und ihnen angeschlossenen Studiobetrieben (z.B. Bavaria Film, Studio Hamburg). Zudem gibt es ein für die Mitbestimmung relevantes Personalmanagement nicht mehr, da:

- auf Selbstständige zurückgegriffen wird, - die befristet Beschäftigten „nur“ abgerechnet werden und

- nur eine extrem kleine Anzahl an dauerhaft beschäftigten Koordinatoren in den Fernsehproduktionsunternehmungen angestellt sind oder die Eigentümer dieser Unternehmungen diese Aufgabe wahrnehmen.

- Zudem übernehmen andere Akteure wie wir gleich am Beispiel der Arbeitskräfteselektion sehen werden - Aufgaben einer Personalabteilung.

\subsection{ARBEITSKRÄFTESELEKTION IN DER TV-CONTENT-PRODUKTION: AKTEURE UND PRAKTIKEN}

Basierend auf der Arbeitskräfteversorgung der fiktionalen Content-Produktion, die heute vor allem Filmhochschulen sicherstellen, werden im Zuge der wechselseitigen Selektion von Fernsehsender und -produzent (dazu genauer Windeler et al. 2000) in einem komplexen Prozess die Arbeitskräfte für Projekte zusammengestellt (Abbildung 1):

\section{ABOVE THE LINE}

Zunächst wird das kreative Personal, das sogenannte Above-the-line-Personal, gebunden. Kreative Fernsehproduzenten, also solche, die eigenständig Inhalte entwickeln, selektieren unter Zugriff auf ihren Arbeitskräftepool zunächst vorläufig Drehbuchautoren, Regisseure und Hauptdarsteller und verknüpfen den Inhalt sowie diese zentralen Arbeitskräfte zu einem Paket (package), das Grundlage für den Ver-

3 Im Normalfall sind TV-Produktionsbetriebe so klein, dass der Schwellenwert für die Bildung von Betriebsräten von fünf Arbeitnehmern, von denen drei wählbar sein müssen, unterschritten wird. Bei einem Großproduzenten beispielsweise koordinieren zehn Arbeitnehmer ca. 360 Arbeitskräfte im Projektnetzwerk in einem Kalenderjahr. 
kauf an einen Fernsehsender ist. Erst nach dem Verkauf des Pakets werden die Arbeitskräfte vom Produzenten vertraglich gebunden. An der Selektion dieser Akteure partizipieren diese individuell und vor dem Hintergrund industriespezifischer Standards. So erfolgt z.B. die Bezahlung von Drehbuchautoren durch die Fernsehproduzenten entsprechend den Entgelten in Gagenregistern der Fernsehsender. Wegen der Partizipation im Schatten solcher Standards betreiben die Akteure zwischen zwei Projekten ein ausgeprägtes Beziehungsmanagement oder lassen - im Fall von Stars - persönliche Manager Beziehungen pflegen. Die erfolgreiche Selektion ist - auch wenn sie häufig personalisiert wird - in einem gewissen Grad kollektiv mitbestimmt, weil Beziehungszusammenhänge, hier sogenannte „starke Beziehungen“" (Burt 1992, S. 25), genutzt werden. Ihr „soziales Kapital“ (Bourdieu 1983) ist die wichtigste Ressource der Arbeitskräfte.

Der Auftrag gebende Fernsehsender übt bei der Auswahl dieser Arbeitskräfte ein vertraglich abgesichertes Vetorecht aus und dehnt auf diese Weise seinen Einfluss in das Unternehmungsnetzwerk aus. Der Fernsehproduzent, bei dem die Mitbestimmung greifen müsste, wird vor dem Hintergrund der Einflussnahme des Fernsehsenders - ähnlich wie in der Just-intime-Produktion in der Automobilindustrie - zu einem „Marionettenarbeitgeber“ (Trümner 1989) und zu einem „Transmissionsriemen“ (Wirth 2010, S. 119) der Fernsehsender, den strategisch platzierten Akteuren in der TV-Content-Produktion. Auch für die Besetzung der Hauptrollen sind die Fernsehsender zentrale Akteure, obwohl diese beim Fernsehproduzenten angestellt werden. Manche Schauspieler werden durch die Entscheidungsrechte des Fernsehsenders zu „A-Schauspielern“(Berufsverband 1, wobei A für den Anfangsbuchstaben des Namens einer wichtigen Redakteurin eines Fernsehsenders steht). Der Zugang (port of entry) zu Hauptrollen verengt sich somit auf wenige Redakteure in dieser Industrie. Das wiederum erleichtert ein spezifisches Beziehungsmanagement, welches vor allem einige Schauspieleragenturen professionell beherrschen, die die Arbeitskräfteselektion im eigenen Interesse und im Interesse ihrer Klienten mitbestimmen. Diese Expertise im Beziehungsmanagement erklärt auch ihren Erfolg und die beherrschende Marktstellung einiger weniger Schauspieleragenturen, die zudem durch die Einsparung von Transaktionskosten gefestigt wird. Im Ergebnis entsteht eine Segmentation der Schauspieler, die - wie von der neueren Personalforschung postuliert - auch Ausdruck von Macht ist. Es gilt z.B. in der Medienregion München die Regel: „Es werden immer die gleichen genommen" (IG Medien 2a) (siehe auch Schmidt 1994).

An die Selektionen dieser für die Akquisition des Projekts zentralen Akteure schließen sich die Auswahl des Regie- und Kamerastabes sowie der Nebendarsteller an. Dabei gilt in der TV-Content-Produktion beispielsweise für die Auswahl der Kameraleute die Regel, dass diese nicht gegen die Regie bestimmt wird, die damit ein Mitbestimmungsrecht bei der Auswahl dieser Arbeitskraft hat. Allerdings variiert dies mit dem Professionalisierungsgrad des Regisseurs und der Art des Produkts. So können Jungregisseure, weil ihre Inhalte auf peripheren Sendeplätzen ausgestrahlt werden und weil man ihre „Handschrift" (Berufsverband 1) kennenlernen möchte, die Selektionen im Regie- und Kamerastab weitaus stärker beeinflussen als erfahrene Regisseure, die in seriellen oder stärker formatierten Produktionen für die Hauptsendezeit aktiv sind. Insofern sind deren künstlerische Freiheiten - im Unterschied zu Theaterregisseuren (s. dazu Haunschild 2002) - in der Regel stark begrenzt.

Die Mitglieder des Kamerastabes wählen die Kameraleute aus, indem sie auf den von ihnen entwickelten und gepflegten Pool von Arbeitskräften zurückgreifen, mit denen sie immer wieder, d.h. von Projekt zu Projekt, zusammenarbeiten. Diese Arbeitskräfte formieren eine „semipermanent workgroup“ (Blair 2001). Der Schlüsselakteur „Kameramann“ bestimmt also vor dem Hintergrund eines Vetorechtes des Fernsehproduzenten und gegebenenfalls des Fernsehsenders die anderen Arbeitskräfte im Kamerastab.

Die Akteure arbeiten dabei in Semipermanent workgroups wiederkehrend zusammen. Sie können so Risiken und Selektionskosten reduzieren sowie Transaktionskosten senkende Routinen ausbilden. In anderen Fällen, z.B. bei der Besetzung von Neben- oder Hauptrollen, haben Regisseure ein Vorschlagsrecht, das einem Beratungsrecht nahekommt, wenn sie ihre „Maskottchen“(Produzent 3a), also ihnen besonders verbundene Schauspieler, bei der Besetzung ins Spiel bringen. Machtvolle Akteure, z.B. Stars, können Arbeitskräfte- selektionen beeinflussen, weil diese Personengruppe mit ihrem Wert für das Produkt über eine wichtige Ressource verfügt. Dementsprechend haben sie ein Vetorecht bei der Besetzung von Kostüm und Maske, denn im Feld gilt für diese Personengruppe die Regel, dass „alles, was man sieht, von ihnen mitbestimmt wird" (Branchenexperte 1a).

Die fortwährende Re-Selektion der Arbeitskräfte in Projektnetzwerken ist an den Erfolg früherer Produktionen und die damit erworbene Reputation geknüpft. So gilt beispielsweise für Regisseure die Regel: "Zwei Quotenflops und man ist draußen" (Berufsverband 1) bzw. allgemeiner für alle Akteure in der TV-Content-Produktion: "you're only as good as your last job“ (Blair 2001).

Für ausführende Fernsehproduzenten, also solche, die vom Fernsehsender entwickelte Inhalte umsetzen, müssen diese Ausführungen modifiziert werden. Durch eine Verschiebung der interorganisationalen Arbeitsteilung gehen Fernsehsender verstärkt dazu über, Inhalte mit Regisseuren oder Drehbuchautoren selbst zu entwickeln. Sie internalisieren quasi den kreativen Part der Inhalteproduktion und stärken so ihre Machtposition gegenüber den Fernsehproduzenten und stellen - im Erfolgsfall - sicher, dass die Inhalte (noch) besser auf ihr Senderprofil zugeschnitten sind. In dieser Konstellation wird der Fernsehproduzent erst ausgewählt, wenn der Inhalt konstituiert ist und die Realisierung ansteht und das dafür benötigte technischhandwerkliche Personal, das sogenannte Below-the-line-Personal, benötigt wird.

\section{BELOW THE LINE}

Der zentrale Akteur für die Selektion des Below-the-line-Personals ist-bei kreativen wie bei ausführenden Fernsehproduzenten - die Herstellungsleitung, die in Absprache mit dem Produzenten die Produktionsleitung auswählt. Diese wiederum selektiert - vor dem Hintergrund eines Vetorechtes der Herstellungsleitung - den Produktionsstab. Dabei greift sie - wie die anderen Heads of Department, also Leiter eines Gewerks, - auf Semi-permanent workgroups zurück, mit denen sie in der Vergangenheit zusammengearbeitet hat. Heads of department sind also Akteure, die das Geschehen trotz fehlender formeller Absicherung vor dem Hintergrund ihrer Beziehungen, ihrer Pools und ihres Beziehungsmanagements 
relativ weitgehend durch ihre Vorschläge und eingespielten Beziehungen mitbestimmen. Sie agieren dabei vor dem Hintergrund eines Vetorechts des Fernsehproduzenten sowie der Herstellungs- und Produktionsleitung. Insofern konstituieren sich - häufig ausgehend von gemeinsamen Zeiten an Filmhochschulen oder in Projekten - im Zuge der Aktualisierung und Institutionalisierung von Beziehungen in Projektnetzwerken entsprechende Beziehungszusammenhänge zwischen Arbeitskräften bzw. Unternehmern und Unternehmungen. In diesen Beziehungszusammenhängen bürgt der Head of department für seine Arbeitskräfte, sodass deren Selektion durch die Produktionsunternehmung wechselseitig voneinander abhängt.

\section{PARTIZIPATION UND ARBEITS- KRÄFTESELEKTION}

Die Mitbestimmung bei der Arbeitskräfteselektion in der TV-Content-Produktion ist vor allem eine im Netzwerkzusammenhang vermittelte Partizipation an den Auswahlentscheidungen ohne formelle Absicherung mit einem geringen Grad an Kollektivität, die sich dementsprechend hinsichtlich der Akteure und Praktiken von den Praktiken in „Normalbetrieben“ unterscheidet:

(1) Die (konzerngebundenen) Fernsehsender dehnen ihren Einfluss in den Netzwerkzusammenhang auch bei der Arbeitskräfteselektion aus: „concentration without centralization" (Harrison 1994).

(2) Der Einfluss auf die Arbeitskräfteselektion hängt von den jeweiligen Ressourcen $\mathrm{ab}$, die die jeweiligen Akteure mobilisieren können, z.B. Beziehungen zu bestimmten Redakteuren oder Reputation.

(3) Heads of Department, die ihre Semipermanent workgroups mobilisieren, bestimmen mit, wer, wie oft und zu welchen Konditionen in Projekten berücksichtigt wird. Einige, so z. B. Regisseure, üben de facto ein Beratungsrecht aus, indem sie Akteure aus ihrem Beziehungszusammenhang vorschlagen.

(4) Die „Personalabteilung“ des Fernsehproduzenten wird zur Abrechnungsstelle (s. dazu auch Yagapen 2007). Sie ist nicht in Auswahlprozesse eingebunden. Akteure jenseits der Unternehmungsgrenze oder
Heads of department treffen relevante Entscheidungen, sodass die Auswahlentscheidungen in die Beziehungszusammenhänge von Fernsehproduzenten weitgehend quasi-externalisiert sind. Sie substituieren auf diese Weise auch die Beteiligung der Fachabteilung in „Normalbetrieben“.

(5) Konfliktlösungen erfolgen nicht durch Arbeitsgerichte oder in Einigungsstellen, sondern in individualisierten Aushandlungsprozessen vor dem Hintergrund professioneller Standards im organisationalen Feld. Die Mitbestimmungsrechte der Arbeitskräfte reduzieren sich zumeist auf Vorschlagsrechte, die in der Regel die Zustimmung anderer Akteure benötigen.

(6) Die Mitbestimmungspraktiken sind auf die Regeln im organisationalen Feld bezogen, die den Akteuren in Projektnetzwerken in Teilen vorgeben, was kompetentes Handeln ist. Diese Professionalitätsnormen substituieren partiell die gesetzlichen Regelungen aus dem Betriebsverfassungsgesetz, ohne jedoch eine gleichwertige Bindung der Praktiken zu erreichen. Es kommt in solchen Bereichen wie der TVContent-Produktion zum Ende der Verrechtlichung industrieller Beziehungen.

(7) „Andere Vertretungsorgane“ (Hertwig 2010) spielen in der TV-Content-Produktion keine Rolle.

(8) Diese Praktiken der Partizipation werden auch in Krisenzeiten in der fiktionalen TV-Content-Produktion fortgeschrieben, weil sie vor allem auf notwendigen Beziehungszusammenhängen basieren. Aber die Bedingungen, unter denen dann Arbeit verausgabt wird, ändern sich für die weniger mächtigen Arbeitskräfte in Medienkrisen dramatisch (Wirth 2010, S. 147ff.).

Die Praktiken der Arbeitskräfteselektion leisten einen zentralen Beitrag zur Lösung der Querschnittsprobleme der Arbeitskräftewirtschaft. So wird beispielsweise durch die Stabilisierung über Semi-permanent workgroups sichergestellt, dass die Akteure anschlussfähige Praktiken ausbilden können und die Lösung des Effektivitätsproblems ermöglicht wird. Indem durch wiederkehrende Selektionen Routinen in der Zusammenarbeit ausgebildet und „Leerstandskosten“ der Ware Arbeitskraft vermieden werden, werden Beiträge zur Lösung des Effizienzproblems erbracht und zugleich die Arbeitskräfte verstärkt unter Konkurrenzdruck gesetzt. In Verbindung mit ihrer fortwährenden Beobachtung im Projekt, im Projektnetzwerk, in der Region sowie in der Industrie werden die Arbeitskräfte zudem diszipliniert. Sie nehmen dementsprechend z. B. überlange Arbeitszeiten in Kauf, um ihre ReSelektion zu befördern (Marrs/Boes 2002; Marrs 2007). Insofern tragen die Praktiken der Arbeitskräfteselektion auch zur Herrschaftssicherung bei. Trotzdem sind die Arbeitskräfte und ihre Interessenvertretungen nicht völlig machtlos. Ich gehe deshalb abschließend auf Ansatzpunkte für Interessenvertretungen ein, mit denen sie die Mitbestimmung fördern können.

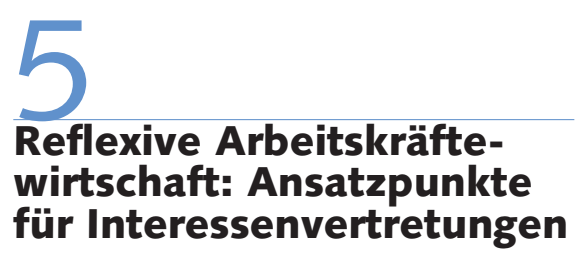

Arbeitskräftewirtschaftliches Handeln hat - auch jenseits der TV-Content-Produktion - einen mehrfach erweiterten Fokus sowie mehr Bedeutung erhalten. Gleichzeitig sorgt die reflexive Arbeitskräftewirtschaft dafür, dass das betriebliche Personalmanagement an Bedeutung einbüßt, weil Akteure im Beziehungszusammenhang und die Arbeitskräfte selbst seine Aufgaben übernehmen und neue Praktiken der Partizipation entstehen. Partizipation erfolgt-das zeigen die empirischen Befunde dieses Beitrags - offenbar auch auf einem anderen Weg. Das Beispiel TV-ContentProduktion gibt erste Hinweise auf Mitbestimmungspraktiken auch in anderen „Betrieben ohne Betriebsrat" (Artus et al. 2006), in denen zwischenbetriebliche Projekte eine hohe Bedeutung haben, zumindest lassen sich Fragen formulieren, die die Analyse der Mitbestimmungspraktiken im jeweiligen Fall befruchten: Wer kann mitbestimmen? Wie wird mitbestimmt? Welche Ressourcen sind dafür bedeutsam?

Trotz der schwierigen Ausgangslage für Interessenvertretungen in der TVContent-Produktion gibt es eine Reihe von Optionen, die sie ergreifen können, um dem demokratischen Potenzial von Mitbestimmung und seinen funktionalistischen Aspekten mehr Bedeutung zu verleihen, ohne aber die Stabilität und Reichweite der Mitbestimmung in den Kernsektoren der Wirtschaft auch nur annähernd zu errei- 
chen. Von Bedeutung ist dabei ein verändertes Zusammenspiel von Interessenvertretung auf staatlicher Ebene, im Betrieb, im Netzwerk und in der Branche:

(1) Aus der Perspektive der Interessenvertretung ist es sinnvoll, das Wissen um die jeweils industriespezifischen Regeln verfügbar zu machen. Das Projekt „connexx. av" der Gewerkschaft ver.di kann als ein solcher industriespezifischer Ansatz interpretiert werden. Regionale Zusammenkünfte, internetbasierte Informationen und - parallel dazu - Initiativen zur Gründung von Betriebsräten, wo dies möglich ist, weisen in die richtige Richtung. Der Austausch und damit die Absprache untereinander, die Gewerkschaftsbildung, kann durch Kooperationen mit anderen Akteuren gefördert werden. Berufsverbände wären trotz einer anderen Logik als Einheitsgewerkschaften ein wichtiger Bündnispartner, mit denen Ressourcen zusammengelegt oder neue generiert werden könnten, um die Mitbestimmung durch Informationsverbreitung (über Selektionspraktiken) zu fördern. Eine engere Kooperation mit Sozialversicherungsträgern, z.B. den Berufsgenossenschaften, könnte zudem die Einhaltung von Arbeitszeitvorschriften verstärken und damit die Arbeitszeit zumindest ein Stück weit mitbestimmen.

(2) Eine Politik des naming and shaming oder öffentlichkeitswirksame Besuche am Set, wie sie zuweilen in Organizing-
Ansätzen propagiert werden, können auf Arbeitskraftnachfrager disziplinierend wirken oder Arbeitgeber in Verhandlungen zwingen, insbesondere wenn es sich dabei um öffentliche Auftraggeber wie öffentlich-rechtliche Fernsehsender oder um Unternehmungen handelt, deren Reputation auf dem Spiel steht. Angesichts der Konkurrenz um kompetente Arbeitskräfte könnten sich auch Ausstrahlungseffekte auf andere Unternehmungen oder Unternehmensnetzwerke ergeben.

(3) Wenn mitbestimmungsrelevante Entscheidungen in Netzwerkzusammenhängen fallen, dann gilt es, den Netzwerkzusammenhang auch als eigenständige Arena der Mitbestimmung zu begreifen. Vergaberichtlinien mit „Sozialklauseln“ für Aufträge an Unternehmungen könnten auch von den relativ machtvollen betrieblichen Interessenvertretungen in öffentlich-rechtlichen Fernsehsendern gefordert und durchgesetzt werden. Sie schützen auf diese Weise ihre relativ privilegierten Arbeitsbedingungen und Mitbestimmungsmöglichkeiten durch eine Solidarität mit anderen und verringern den Druck in Richtung „Outsourcing“.

(4) Die Bezugnahme auf Professionalitätsnormen durch die Arbeitskräfte und deren Verbreitung, indem beispielsweise an (Film-) Hochschulen und an den zahlreichen Treffpunkten der Branche die Gewerkschaften präsent(-er) wären, könnte die Mitbestimmung der Arbeitskräfte stärken, weil viele Praktiken in der
TV-Content-Produktion auf der nicht mehr hinterfragten Fortschreibung einer bestimmten Praxis beruhen. Es geht aber auch um die Mitgestaltung von Sicht- und Legitimationsweisen in der Interaktion im Feld der TV-Content-Produktion, die die Grundlage von Professionalitätsnormen sind. Gewerkschaften könnten also auch als „institutional entrepreneur“ (DiMaggio 1988) aktiv werden, die interessengeleitet Institutionen etablieren.

(5) Im Zuge neo-korporatistischer Arrangements wären unter Berücksichtigung von Interessendivergenzen auf der Arbeitskraftnachfragerseite auch die Kontextbedingungen in der TV-Content-Produktion zu beeinflussen, z. B. durch eine Veränderung des Urheberrechts. Zudem müsste im Zuge einer Rechtsreform „das Recht der Netzwerke“ (Lange 1998) weiterentwickelt werden, um die Grundlagen von Wirtschaft, Sozialstaat und Mitbestimmung der Existenz dieses Akteurs anzupassen.

Für die weitere Entwicklung der Mitbestimmung wird mitentscheidend sein, ein erweitertes Verständnis für Mitbestimmungspraktiken zu erlangen, das auch andere Partizipationsformen in mitbestimmungsfreien Zonen entschlüsseln kann. Dies wäre eine wichtige Voraussetzung für die Bearbeitung und Schließung von Mitbestimmungslücken sowie für die Verringerung der Mitgliederrekrutierungsprobleme der Gewerkschaften.

\section{LITERATUR}

Artus, I./Böhm, S./Lücking, S./Trinczek, R. (Hrsg.) (2006): Betriebe ohne Betriebsrat, Frankfurt a. M./New York

Barley, S. R./Kunda, G. (2004): Gurus, hired guns, and warm bodies. Itinerant experts in a knowledge economy, Princeton/Oxford

Blair, H. (2001): "You're only as good as your last job": the labour process and the labour market in the British film industry, in: Work, Employment and Society 15 (1), S. 149-169

Bourdieu, P. (1983): Ökonomisches Kapital, kulturelles Kapital, soziales Kapital, in: Kreckel, R. (Hrsg.): Soziale Ungleichheiten. Soziale Welt, Sonderband 2, S. 183-198

Burt, R. S. (1992): Structural holes. The social structure of competition, Cambridge, Mass.
Crimmann, A./Ziegler, K./Ellguth, P./Kohaut, S./Lehner, F. (2009): Forschungsbericht zum Thema "Arbeitnehmerüberlassung “, Nürnberg DiMaggio, P. J. (1988): Interest and agency in institutional theory, in: Zucker, L. (Hrsg.): Institutional patterns and organizations, Cambridge, S. 3-21

DiMaggio, P. J./Powell, W. W. (1983): The iron cage revisited: Institutional isomorphism and collective rationality in organizational fields, in: American Sociological Review 48 (2), S. 147-160

Ellguth, P./Kohaut, S. (2011): Tarifbindung und betriebliche Interessenvertretung. Aktuelle Ergebnisse aus dem IAB-Betriebspanel 2010, in: WSI-Mitteilungen 63 (5), S. 242-247, http://www.boeckler.de /119_113621.html 
Giddens, A. (1984): The constitution of society, Cambridge

Granovetter, M. (1973): The strength of weak ties, in: American Journal of Sociology 78 (6), S. 1360-1380

Haak, C. (2008): Wirtschaftliche und soziale Risiken auf den Arbeitsmärkten von Künstlern, Wiesbaden

Harrison, B. (1994): Lean and mean. The changing landscape of corporate power in the age of flexibility, New York

Haunschild, A. (2002): Das Beschäftigungssystem Theater, in: Zeitschrift für Personalforschung 16 (4), S. 577-598

Hertwig, M. (2009): "Kollegiale Arbeitnehmerüberlassung“ - Chancen und Probleme des zwischenbetrieblichen Personaltransfers auf Basis von Tarifverträgen, in: Industrielle Beziehungen 16 (3), S. 254-272

Hertwig, M. (2010): Die Praxis "Anderer Vertretungsorgane": Form, Funktion und Wirksamkeit, Berlin

Kißler, L./Greifenstein, R./Schneider, K. (2011): Die Mitbestimmung in der Bundesrepublik Deutschland. Eine Einführung, Wiesbaden

König, S. (2005): Human Resource Management, Personalauswahl und Theorien industrieller Beziehungen. Interaktionskulturen aus einer Negotiated Order-Perspektive, München/Mering

Lange, K. W. (1998): Das Recht der Netzwerke, Heidelberg

Marrs, K. (2007): Zwischen Leidenschaft und Lohnarbeit. Ein arbeitssoziologischer Blick hinter die Kulissen von Film und Fernsehen, Berlin Marrs, K./Boes, A. (2002): Schatten im Scheinwerferlicht - Arbeitsund Leistungsbedingungen in der Audio-visuellen Medienbranche, in: WSI-Mitteilungen 55 (9), S. 517-523

Nienhüser, W. (1999): Personalwirtschaftliche Konsequenzen der Externalisierung von Arbeit - Die Bauwirtschaft als prototypisches Beispiel, in: Sydow, J./Wirth, C. (Hrsg.): Arbeit, Personal und Mitbestimmung in Unternehmungsnetzwerken, München/Mering, S. 143-173

Ortmann, G. (1995): Formen der Produktion. Organisation und Rekursivität, Opladen

Promberger, M. (2006): Leiharbeit - Flexibilität und Prekarität in der betrieblichen Praxis, in: WSI-Mitteilungen 59 (5), S. 263-269

Schmidt, S. (1994): "Es muss ja nicht gleich Hollywood sein“. Die Produktionsbedingungen des Fernsehspiels und die Wirkungen auf seine Ästhetik, Berlin
Staehle, W. H. (1989): Human Resource Management und Unternehmungsstrategie, in: Mitteilungen aus der Arbeitsmarkt- und Berufsforschung 22 (3), S. 388-396

Sydow, J./Windeler, A. (1999): Projektnetzwerke: Management von (mehr als) temporären Systemen, in: Engelhard, J./Sinz, E. J. (Hrsg.): Kooperation im Wettbewerb. Neue Formen und Gestaltungskonzepte im Zeichen von Globalisierung und Informationstechnologie, Wiesbaden, S. 211-235

Trümner, R. (1989): Just-in-time-Produktion - eine Herausforderung an die betriebliche Mitbestimmung, in: Doleschal, R./Klönne, A. (Hrsg.): Just-in-time-Konzepte und Betriebspolitik, Düsseldorf, S. 166-179 Weinkopf, C. (1996): Arbeitskräftepools, München/Mering Windeler, A./Lutz, A./Wirth, C. (2000): Netzwerksteuerung durch Selektion - Die Produktion von Fernsehserien in Projektnetzwerken, in: Sydow, J./Windeler, A. (Hrsg.): Steuerung von Netzwerken, Opladen/Wiesbaden, S. 178-205

Windeler, A./Wirth, C. (2004): Arbeitsregulation in Projektnetzwerken: Eine strukturationstheoretische Analyse, in: Industrielle Beziehungen 11 (4), S. 295-319

Windeler, A./Wirth, C./Sydow, J. (2001): Die Zukunft in der Gegenwart erleben. Arbeiten in Projektnetzwerken der Fernsehproduktion, in: Arbeitsrecht im Betrieb 22 (1), S. 12-18

Wirth, C. (1999): Franchising- und franchisingähnliche Systeme - Das Ende von Interessenvertretung?, in: Sydow, J./Wirth, C. (Hrsg.): Arbeit, Personal und Mitbestimmung in Unternehmungsnetzwerken, München/Mering, S. 233-256

Wirth, C. (2010): Reflexive Arbeitskräftewirtschaft. Strukturation, Projektnetzwerke und TV-Content-Produktion, München/Mering Wirth, C./Sydow, J. (2004): Hierarchische Heterarchien - heterarchische Hierarchien. Zur Differenz von Konzern- und Netzwerksteuerung in der Fernsehproduktion, in: Sydow, J./Windeler, A. (Hrsg.):

Organisation der Content-Produktion, Wiesbaden, S. 125-147 Yagapen, M. (2007): Filmgeschäftsführung, Konstanz 